\title{
Catheter-based photoacoustic endoscope for use in the instrument channel of a clinical video endoscope
}

Joon-Mo Yang, Chiye Li, Ruimin Chen, Qifa Zhou, K. Kirk Shung, et al.

Joon-Mo Yang, Chiye Li, Ruimin Chen, Qifa Zhou, K. Kirk Shung, Lihong V. Wang, "Catheter-based photoacoustic endoscope for use in the instrument channel of a clinical video endoscope," Proc. SPIE 9323, Photons Plus Ultrasound: Imaging and Sensing 2015, 93230Y (11 March 2015); doi: $10.1117 / 12.2080094$

SPIE. Event: SPIE BiOS, 2015, San Francisco, California, United States 


\title{
Catheter-based photoacoustic endoscope for use in the instrument channel of a clinical video endoscope
}

\author{
Joon-Mo Yang ${ }^{1,3}$, Chiye Li ${ }^{1,3}$, Ruimin Chen ${ }^{2}$, Qifa Zhou ${ }^{2}$, K. Kirk Shung ${ }^{2}$, and Lihong V. Wang ${ }^{1 *}$ \\ ${ }^{1}$ Optical Imaging Laboratory, Department of Biomedical Engineering, Washington University in St. \\ Louis, One Brookings Drive, Campus Box 1097, St. Louis, Missouri 63130, USA \\ ${ }^{2}$ Ultrasonic Transducer Resource Center, Department of Biomedical Engineering, University of \\ Southern California, 1042 Downey Way, University Park, Los Angeles, California 90089, USA
}

\begin{abstract}
We have successfully developed a fully-sheathed, flexible shaft-based, mechanical scanning photoacoustic endoscopy (PAE) system for imaging the human gastrointestinal tract via the instrument channel of a clinical video endoscope. The endoscopic system uses a single element ultrasonic transducer and flexible shaft-based proximal actuation mechanism, and it has a $2.5 \mathrm{~m}$ long and $3.2 \mathrm{~mm}$ diameter catheter section, which can be accommodated in the $3.7 \mathrm{~mm}$ diameter instrument channel of a clinical video endoscope. Here, we demonstrate the intra-instrument channel workability and in vivo imaging capability of the PAE system.
\end{abstract}

Keywords: Photoacoustic endoscopy, flexible shaft, catheter-based photoacoustic endoscope, instrument channel, gastrointestinal endoscopy, rabbit esophagus, in vivo imaging.

\section{INTRODUCTION}

Barrett's esophagus [1-3] is the most representative esophageal disease caused by gastro-esophageal reflux, and it is regarded as a precursor of esophageal cancer, such as adenocarcinoma. To detect the symptoms of abnormal change in Barrett's esophagus, there have been many efforts to develop novel endoscopic techniques, but no effective in situ diagnostic method has been developed so far. However, in recent years, several papers have suggested that the microvasculature pattern in Barrett's esophagus can be important diagnostic information for the early detection of esophageal cancer [4-8]. At present, endoscopic ultrasound (EUS) [9-11] is the only clinically-available tomographic endoscopic tool used to diagnose many diseases in the gastrointestinal (GI) tract, including esophageal diseases. However, due to the limited contrast mechanism, it cannot provide microvasculature details of the esophagus. Thus, to image microvasculature, several new endoscopic imaging techniques, such as narrow band imaging [4-6], confocal endoscopy [8], and endoscopic optical coherence tomography (OCT) [12,13], have been intensively developed in recent years. However, again due to the contrast mechanism, their vasculature imaging capability is not as high as that of photoacoustic endoscopy (PAE) $[14,15]$, which can provide high-resolution vasculature images and functional information without using any contrast agent, as can photoacoustic (PA) tomography [16-50].

So far, for gastrointestinal (GI) tract imaging, we developed multiple PAE systems [44-50] and demonstrated their in vivo imaging capability through animal experiments. However, we recognized that, although the developed imaging probes could be utilized for some clinical applications, such as lower GI tract [47] or urogenital system imaging [49], they cannot be utilized for human esophagus imaging. To image the human esophagus, it is essential to use a video endoscope for image guidance to approach the target safely. However, the previously developed endoscopic probes [45, 47] cannot easily pass through the instrument channels of clinical video endoscopes, which typically are $2.8 \mathrm{~mm}$ or 3.7 $\mathrm{mm}$ in diameter, because their rigid distal section lengths are too long. Thus, in this study, to image the human upper GI tract via the instrument channel of a clinical video endoscope, we developed a new endoscopic system, called a catheter-based photoacoustic endoscope [50]. The system features a single element ultrasonic (US) transducer and a

${ }^{3}$ These authors contributed equally to this work. "Corresponding author: lhwang@biomed.wustl.edu

Photons Plus Ultrasound: Imaging and Sensing 2015, edited by Alexander A. Oraevsky, Lihong V. Wang

Proc. of SPIE Vol. 9323, 93230Y · (C) 2015 SPIE · CCC code: 1605-7422/15/\$18 doi: $10.1117 / 12.2080094$

Proc. of SPIE Vol. $932393230 Y-1$ 
flexible shaft-based proximal actuation mechanism which is commonly utilized in many clinical EUS miniprobes [51], and uses a rotary junction-based light coupling and delivery method that is typically employed in many endoscopic OCT probes [12, 13, 52-55]. For the prototype imaging system, most important achievement is that all the mechanical, optical, and acoustical components are fully encapsulated in a $3.2 \mathrm{~mm}$ diameter and $\sim 2.5 \mathrm{~m}$ long plastic sheath, which enables its free insertion into the instrument channel of a clinical video endoscope. In this paper, we introduce the catheter-based PAE system, discuss related technical issues in imaging the human esophagus, and consider future system improvement directions.

\section{MATERIALS AND METHODS}

\subsection{Catheter-based photoacoustic endoscope}

In Figure 1(a), we schematically present the structure and operating principles of the endoscopic system. And, as shown in the prototype system photo [Fig. 1(b)], we achieved a $3.2 \mathrm{~mm}$ probe diameter with a $\sim 16 \mathrm{~mm}$ long rigid distal section, so it can easily fit the $3.7 \mathrm{~mm}$ diameter instrument channel of a clinical video endoscope. To reduce the rigid distal section length and improve the overall flexibility, we utilized a flexible shaft (TC+2113-70-2600-01, Asahi Intecc) to transmit mechanical torque from the step motor installed in the proximal actuation unit to the scanning mirror installed in the rigid distal section. Also, we delivered the laser beam through two identical multimode optical fibers (0.22 NA, $365 \mu \mathrm{m}$ core dia., BFL22-365, Thorlabs), which are coupled at a rotary junction. During mechanical scanning, the optical fiber installed in the flexible shaft rotates, but the other optical fiber remains static. So, while the flexible shaft and scanning mirror rotate, we send out laser pulses guided from a light source to the target tissue and detect generated PA signals using the US transducer $\left(40 \mathrm{MHz}, 1.7 \mathrm{~mm}\right.$ aperture, $f=5 \mathrm{~mm}, \mathrm{NA}=0.17, \mathrm{LiNbO}_{3}$ ) installed at the tip of the probe. We press-focused the transducer to achieve the focusing capability. Press-focusing [56] is superior to the previous lens-based focusing [44], which affixed a plastic acoustic lens to the surface of a flat transducer, and thus press-focusing yields higher sensitivity without the acoustic attenuation caused by the plastic lens.

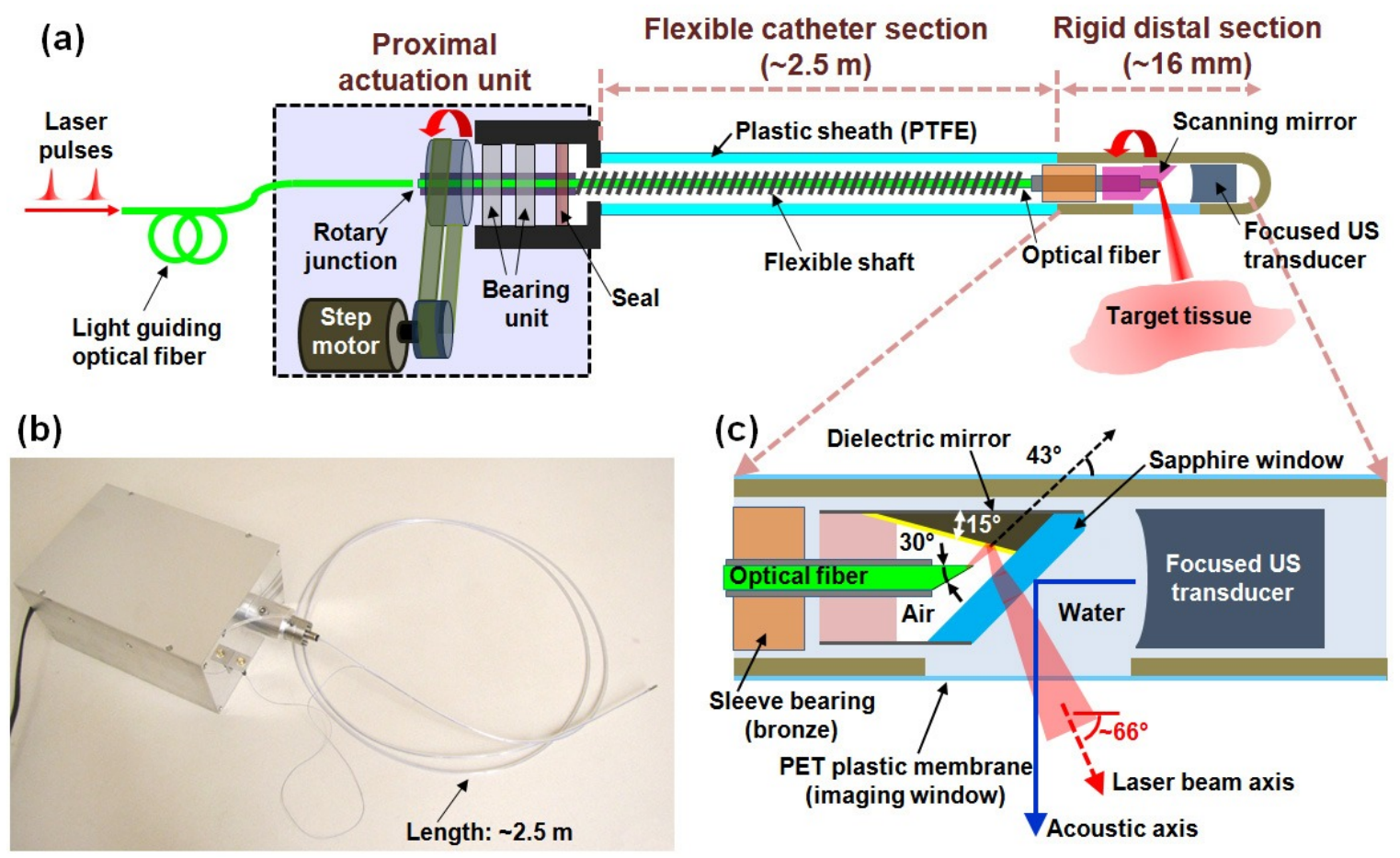

Figure 1. (a) Schematic of the catheter-based PAE system. (b) Photo of the prototype catheter-based PAE system. (c) Schematic showing the configuration of optical and acoustic components at the rigid distal section. 
In this system, to illuminate the target tissue as perpendicularly as possible with the given probe configuration, we designed the optical and acoustical unit at the distal section as shown in Figure 1(c). Since we utilized a multimode optical fiber with a large core diameter $(365 \mu \mathrm{m})$ and a high optical numerical aperture $(0.22)$, the laser beams exiting the optical fiber were also correspondingly large. Thus, to deliver the entire large diameter laser beam, we polished the fiber tip to a $\sim 30^{\circ}$ angle as with the method reported by Karpiouk et al. [36] and placed a tiny dielectric mirror (altered from \#45-596, Edmund) as shown in the diagram. Once the laser beam exits the fiber tip, it is sent to the dielectric mirror, reflected, and finally delivered to the target tissue after passing through the sapphire window (altered from \#43627 , Edmund) and an optically clear polyethylene terephthalate (PET) plastic membrane ( $\sim 3 \mathrm{~mm}$ inner diameter, $\sim 25$ $\mu \mathrm{m}$ wall thickness, 103-0380, Advanced Polymers). Here, we chose sapphire as the window material because it can effectively reflects incoming acoustic waves.

With the presented optical design, we could achieve an optical illumination angle of $66^{\circ}$, which creates a directional mismatch between the optical illumination and acoustic detection. To show that we can deliver adequate laser energy to the imaging plane of the transducer despite the mismatch, we performed a Monte Carlo simulation [57] using the beam profile information at the output and the typical optical property values of human mucosa [58]. The simulation showed that, although the energy is lower than that in the major beam location by one order of magnitude, adequate laser energy can be delivered to the imaging plane of the transducer.

As the light source of the endoscopic system, we utilized a Q-switched diode-pumped Nd:YVO4 laser (INNOSLAB IS811-E, EdgeWave) which provides a 532-nm laser wavelength with an $\sim 10 \mathrm{~ns}$ pulse duration. We set the laser energy to $\sim 0.25 \mathrm{~mJ}$, which yielded an optical fluence of lower than $3 \mathrm{~mJ} / \mathrm{cm}^{2}$ (i.e., $15 \%$ of the ANSI safety limit for allowable skin laser fluence [59]). Once a laser pulse is delivered to target tissue, it induces PA waves which then can be detected by the US transducer and recorded by a data acquisition (DAQ) card (NI PCI-5124, National Instruments) after being amplified by an US pulser-receiver (5072PR, Panametrics). To trigger the laser and DAQ card as well as the step motor's driver circuits, we employed a function generator (SFG-2110, GWInstek) that provides TTL signals with a continuously variable frequency. In performing endoscopic imaging, we used $400 \mathrm{~A}$-lines for one full B-scan, which yields an angular step size of $0.9^{\circ}$, considering the theoretical transverse resolution of the endoscope.

In implementing the probe based on the configuration presented in Figure 1(a), there were two challenging issues. The first one was to provide an appropriate route for the signal wire $(0.44 \mathrm{~mm}$ dia., $50 \Omega$, 40232-1-600-S-00000, Hitachi Cable Manchester) of the transducer. Since the optical fiber and flexible shaft occupy most of the inner space of the plastic catheter (PTFE, wall thickness: $0.80 \mathrm{~mm}$ ) and the transducer is located at the tip of the endoscope, we shaved one side of the plastic catheter and the stainless steel housing, carefully aligned the signal wire, and finally fixed it in place using two very-thin medical-grade PET heat shrink tubes (\#103-0147 for the $2.5 \mathrm{~m}$ long flexible body section and \#103-0380 for the $16 \mathrm{~mm}$ long rigid distal section, Advanced Polymers). By eliminating crevices, the heat shrink tubes make it easier to sterilize the probe surface, and they can be easily replaced whenever necessary. In summary, we deliver laser pulses through a rotating optical fiber coaxially placed inside the flexible shaft, and transmit electric signals from an US transducer through a static signal wire that is affixed on the outer surface of a plastic sheath.

The second challenge was to provide acoustic matching within the inner cavity of the mechanically scanning endoscopic probe. We chose deionized water because it exhibits high optical transparency and low acoustic attenuation. However, it gradually corrodes metallic components. Thus, we designed the endoscope so that we could inject water before each experiment and drain it out afterwards. To inject water, we immerse the tip of the probe in a water tank and connect the other end to a vacuum system which sucks water through the injection port at the distal tip of the probe. After the probe is filled, we close the injection port, using a sealing screw with an O-ring. If, on visual inspection under a $\sim 20 \times$ microscope, bubbles are seen inside the cavity, the same procedure is repeated until no bubbles appear.

More detailed information regarding the acoustic matching is described in our recent report [50]. However, we recognized that water injection without bubbles requires a very long time. Thus, in our more recent work, we built three alternative endoscopic probes as shown in Figure 2(a). The only difference between the three new probes [Fig. 2(a)] and the prototype probe [Fig. 1(b)] is that their working distances (from the endoscopes' plastic membrane surfaces) were reduced from $2 \mathrm{~mm}$ to $1 \mathrm{~mm}$ to increase their transverse resolutions. In performing the proposed human esophagus imaging experiment, our plan is to prepare all the three endoscopic probes prior to imaging and chose the probe with the fewest bubbles, which then could be connected to the proximal actuation unit as shown in Figure 2(b). 
(a)

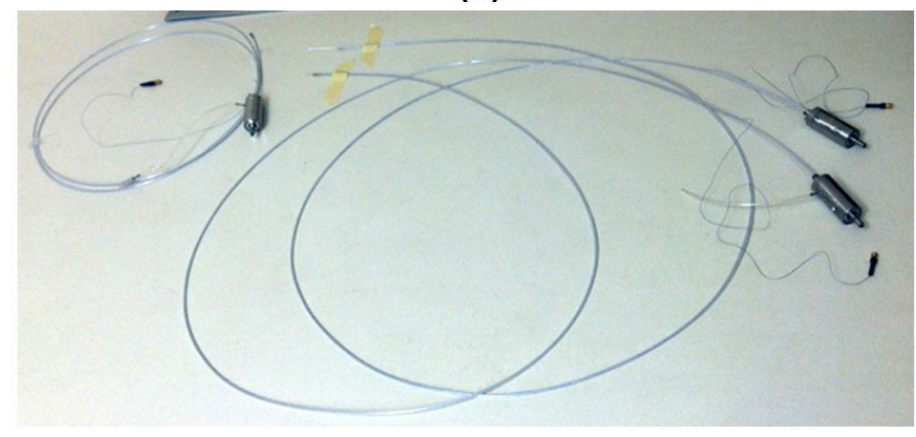

(b)

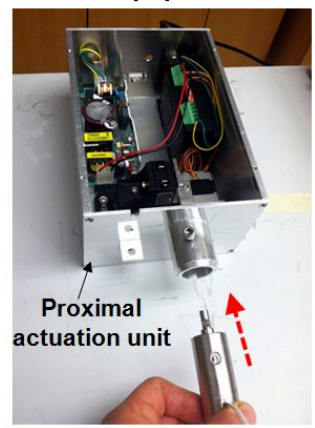

Figure 2. Photos of (a) the three alternative probes and (b) connecting a probe to the proximal actuation unit of the catheter-based PAE system.

\section{SYSTEM VALIDATIONS}

\subsection{Quantification of basic system performances}

With the employed laser system, it was theoretically possible to achieve $5 \mathrm{kHz}$ A-line acquisition rate, which would correspond to a $12 \mathrm{~Hz}$ B-scan rate. However, the actual B-scan speed of the prototype system was limited to only $5 \mathrm{~Hz}$ (i.e., an A-line acquisition rate of $2 \mathrm{kHz}$ ) due to the limited capacity of the step motor and the high mechanical friction generated between the $2.5 \mathrm{~m}$ long flexible shaft and the plastic catheter.

After a basic mechanical test, we imaged a $20-\mu \mathrm{m}$ thick tungsten wire fixed at the focal distance of the endoscope to quantify the spatial resolution (underwater imaging). From the tungsten wire, we acquired $200 \mathrm{~B}$-scan images to determine the resolutions more accurately by averaging multiple B-scan images pixel by pixel. The radial resolution and transverse resolution were $\sim 154 \mu \mathrm{m}$ and $\sim 160 \mu \mathrm{m}$, respectively. The radial resolution was worse than the theoretical value because there was an electrical impedance mismatch between the transducer and the amplifier. However, the transverse resolution was close to the theoretical value. From this experiment, we also quantified the rotational stability (mechanical jitter) of the scanning mirror. By analyzing the lateral fluctuation of the tungsten wire in the acquired 200 B-scan images, we could determine that the scanning mirror exhibited a root mean square (rms) fluctuation of $\sim 2.2$ degrees in angle, which became greater when the catheter was bent.

\subsection{Demonstration of the intra-instrument channel workability}

To show the endoscope's intra-instrument channel workability, we utilized a clinical video endoscope (13911 NKS, Karl Storz) with a $3.7 \mathrm{~mm}$ diameter instrument channel. As shown in Figure 3, the probe could pass through the sharply-bent entry port of the instrument channel and perform a series of angulations along with the video endoscope. Also, we could retract the probe from the instrument channel uneventfully.
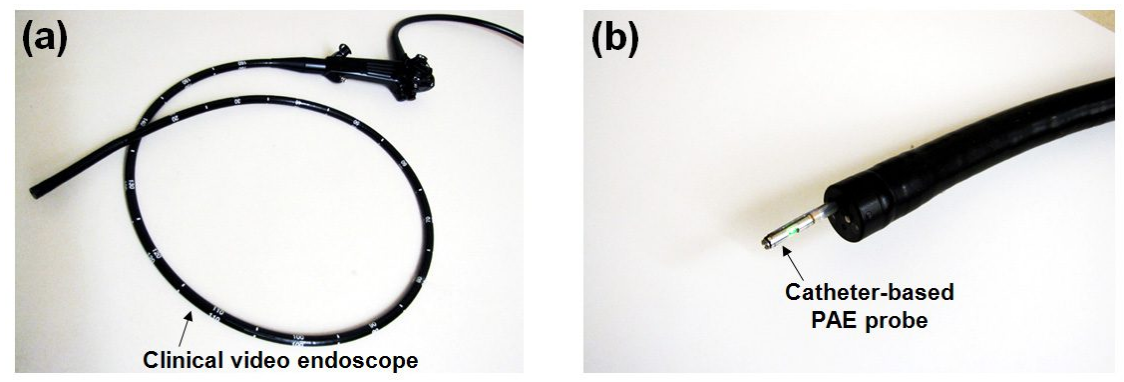

Figure 3. Photos of a clinical video endoscope (a) and the catheter-based PAE probe positioned projecting from the 3.7-mm instrument channel of a clinical video endoscope (b). 
Thus, in a real human imaging experiment, we can adjust the longitudinal position of the probe once it comes out through the exit port of the video endoscope. We can approach target organ by performing angulations of the video endoscope and start to record B-scan images with a frame rate of $5 \mathrm{~Hz}$.

\subsection{In vivo demonstration}

To demonstrate the system's in vivo imaging capability, we imaged the upper esophagus of a New Zealand white rabbits ( $\sim \mathrm{kg}$, Harlan Laboratories). Since the diameter $(\sim 13 \mathrm{~mm})$ of the video endoscope is much larger than the typical diameter $(\sim 5 \mathrm{~mm})$ of a rabbit's esophagus, we utilized only the PAE probe for this experiment. After the anesthesia of the animal and setting up necessary devices for the maintenance of anesthesia, we placed the rabbit on an inclined stage $\left(\sim 10^{\circ}\right)$ in supine position and inject water into the esophagus for acoustic coupling. We then inserted the endoscopic probe through the mouth, advanced it approximately $25 \mathrm{~cm}$ (to the point at which the probe could no longer be gently advanced), and performed a pullback C-scan over a $\sim 6 \mathrm{~cm}$ range during constant pullback translation of the probe by a motorized translation stage at a speed of $\sim 150 \mu \mathrm{m} / \mathrm{s}$, which yields a longitudinal spacing of $\sim 30 \mu \mathrm{m}$. Throughout the experiment, the rabbit's anesthesia level and vital signs were continuously monitored. After the experiment, the rabbit was euthanized by an overdose of sodium pentobarbital $(150 \mathrm{mg} / \mathrm{kg})$ injected in the marginal ear vein. More detailed experimental procedures are fully described in ref. 50. All procedures in the animal experiments followed the protocol approved by the Institutional Animal Care and Use Committee at Washington University in St. Louis.

In Figure 4, we present representative B-scan images acquired from the animal, with a field-of-view (FOV) of $160^{\circ}$ and a $5.4 \mathrm{~mm}$ imaging depth. Since the PA A-line raw data includes negative values, i.e., they are bipolar, we applied the Hilbert transform to extract the envelope of the signal, and we present the Hilbert-transformed images on a logarithmic color scale to more clearly show the image structures at large depths. As shown in the B-scan images, the endoscope could provide cross-sectional images showing the entire wall of the esophagus (typical thickness: $\sim 400-600 \mu \mathrm{m}$ ) and other structures, such as blood vessels (the arrows), distributed around the esophagus. However, due to the mismatch between the laser illumination and acoustic detection, we could not detect signals when the probe's distance to the esophageal surface increased beyond approximately more than $1.5 \mathrm{~mm}$ (see the dotted lines). This result implies that the endoscope must used in direct contact mode, or nearly so.

(a)

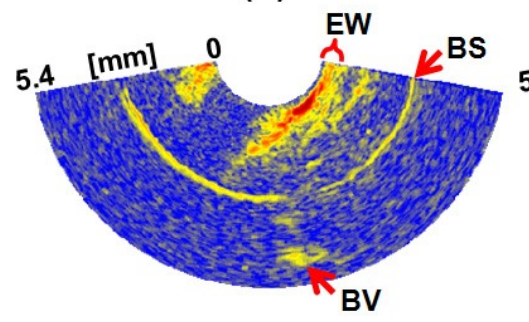

(b)
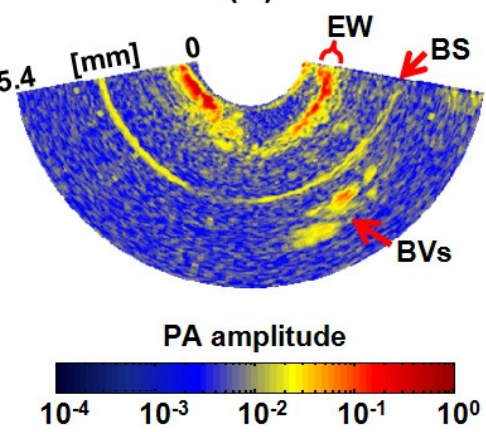

(c)

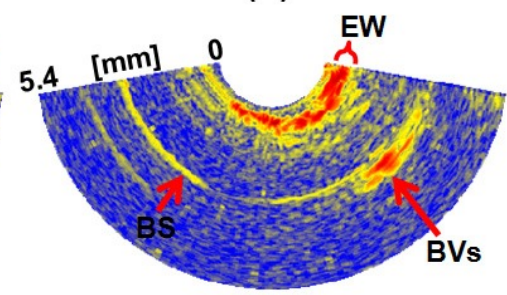

Figure 4. Representative PAE B-scan images of a rabbit esophagus acquired in vivo, shown on a logarithmic color scale. The images cover an $\sim 14 \mathrm{~mm}$ diameter (i.e., $\sim 5.4 \mathrm{~mm}$ radial imaging depth) and $\sim 160^{\circ}$ angular FOV. The left-hand side and lower portion of each image correspond to the left and the dorsal sides of the animal, respectively. BS, bounceback signals; BV, blood vessel; EW, esophageal wall.

Using the acquired C-scan data, we also tried to produce a radial maximum amplitude projection or a volumetric image. However, we could not acquire decipherable images because the nonuniform rotational distortion was very serious. This distortion did not occur with the previous micromotor-based endoscopes [45, 47].

\section{DISCUSSION}

In this study, we developed the first fully-sheathed, flexible shaft-based PA endoscope, with a $3.2 \mathrm{~mm}$ diameter and 2.5$\mathrm{m}$ long catheter section. The endoscope can be utilized in the $3.7-\mathrm{mm}$ diameter instrument channel of a standard clinical 
video endoscope. By employing a mechanical source installed at the proximal part of the imaging probe, we could greatly reduce the rigid distal section length of the imaging probe, to $\sim 16 \mathrm{~mm}$, about two times shorter than in previous micromotor-based endoscopic probes [45, 47]. And, for the prototype imaging system, we achieved a B-scan imaging speed of $5 \mathrm{~Hz}$, which could be further improved by employing a more powerful motor. We quantified the imaging performance of the system and demonstrated its intra-instrument channel workability and in vivo imaging capability.

To make the catheter-based PA endoscope more clinically useful, we recognize that the system needs to be improved in several aspects. The most important task is to increase the illumination angle to make the laser beam overlap the imaging plane of the transducer more closely. Thus, the dead signal zone shown in Figure 4 will disappear. The second improvement is to develop a new probe with a medical balloon $[12,13,53,54]$ to improve the acoustic coupling efficiency between the probe and target tissue. In addition, we are considering how to implement a dual wavelengthbased catheter endoscopic system for $\mathrm{sO}_{2}$ measurement, and to increase the probe's flexibility and scanning speed. If the imaging speed can be improved to a real-time image frame rate (typically $\sim 30 \mathrm{~Hz}$ ) and the catheter probe can be combined with a balloon, volumetric images can be acquired without motion artifacts. Last, we recently demonstrated the feasibility of optical-resolution photoacoustic endomicroscopy using a built-in scanning mirror and micromotorbased mechanical scanning mechanism [60], and we will seek to extend this method to a new catheter-based PA endoscope. The optical focusing would increase the spatial resolution for imaging microvasculature.

\section{CONCLUSION}

In this study, we showed the feasibility of a catheter-based photoacoustic endoscope system by implementing a prototype PAE system that has a 3.2-mm diameter and $2.5-\mathrm{m}$ long catheter section. With the prototype, we achieved a B-scan frame rate of $\sim 5 \mathrm{~Hz}$ and an A-line acquisition rate of $2 \mathrm{kHz}$. We demonstrated its endoscopic imaging ability through an in vivo rabbit esophagus imaging experiment. Most notably, the 3.2-mm diameter catheter was small enough to pass through the $3.7-\mathrm{mm}$ diameter of a standard instrument channel in a clinical video endoscope. With this endoscope, we will progress to a proposed Barrett's esophagus imaging study.

\section{ACKNOWLEDGMENT}

We thank Prof. James Ballard for his attentive reading of the manuscript, and Dr. Konstantin Maslov for useful discussions on the system design. We also thank Lisa Andrews-Kaminsky and Jenny Kalishman for helping with the animal experiment. This work was sponsored in part by National Institutes of Health grants R01 CA157277, DP1 EB016986 (NIH Director's Pioneer Award), R01 EB016963, and R01 CA159959. L.W. has a financial interest in Microphotoacoustics, Inc. and Endra, Inc., which, however, did not support this work.

\section{REFERENCES}

[1] Spechler, S. J., Sharma, P., Souza, R. F., Inadomi, J. M. and Shaheen, N. J., "American Gastroenterological Association Technical Review on the Management of Barrett's Esophagus," Gastroenterology 140(3), e18-e52 (2011).

[2] Wilson, B. C., "Detection and treatment of dysplasia in Barrett's esophagus: a pivotal challenge in translating biophotonics from bench to bedside," J. Biomed. Opt. 12(5), 051401 (2007).

[3] Sato, F., Jin, Z., Schulmann, K., Wang, J., Greenwald, B. D., Ito, T., Kan, T., Hamilton, J. P., Yang, J., Paun, B., David, S., Olaru, A., Cheng, Y., Mori, Y., Abraham, J. M., Yfantis, H. G., Wu, T. T., Fredericksen, M. B., Wang, K. K., Canto, M., Romero, Y., Feng, Z. and Meltzer, S. J, "Three-tiered risk stratification model to predict progression in Barrett's esophagus using epigenetic and clinical features," PLoS One 3(4), e1890 (2008).

[4] Kara, M. A., Ennahachi, M., Fockens, P., ten Kate, F. J. and Bergman, J. J, "Detection and classification of the mucosal and vascular patterns (mucosal morphology) in Barrett's esophagus by using narrow band imaging," Gastrointest. Endosc. 64(2), 155-166 (2006).

[5] Singh, R., Anagnostopoulos, G. K., Yao, K., Karageorgiou, H., Fortun, P. J., Shonde, A., Garsed, K., Kaye, P. V., Hawkey, C. J. and Ragunath, K., "Narrow-band imaging with magnification in Barrett's esophagus: validation of a simplified grading system of mucosal morphology patterns against histology," Endoscopy 40(6), 457-463 (2008). 
[6] Lee, M. M. and Enns, R., "Narrow band imaging in gastroesophageal reflux disease and Barrett's esophagus," Can. J. Gastroenterol. 23(2), 84-87 (2009).

[7] Thekkek, N., Pierce, M. C., Lee, M. H., Polydorides, A. D., Flores, R. M., Anandasabapathy, S. and RichardsKortum, R. R., "Modular video endoscopy for in vivo cross-polarized and vital-dye fluorescence imaging of Barrett's-associated neoplasia," J. Biomed. Opt. 18(2), 26007 (2013).

[8] Becker, V., Vieth, M., Bajbouj, M., Schmid, R. M. and Meining, A., "Confocal laser scanning fluorescence microscopy for in vivo determination of microvessel density in Barrett's esophagus," Endoscopy 40(11), 888-891 (2008).

[9] Dietrich, C., [Endoscopic Ultrasound: An Introductory Manual and Atlas], Thieme, New York (2011).

[10] Hawes, R. H. and Fockens, P., [Endosonography], Saunders Elsevier, Canada (2006).

[11] Shami, V. M. and Kahaleh, M., [Endoscopic Ultrasound], Humana Press, New York (2010).

[12] Yun, S. H., Tearney, G. J., Vakoc, B. J., Shishkov, M., Oh, W. Y., Desjardins, A. E., Suter, M. J., Chan, R. C., Evans, J. A., Jang, I. K., Nishioka, N. S., De Boer, J. F. and Bouma, B. E., "Comprehensive volumetric optical microscopy in vivo," Nat. Med. 12(12), 1429-1433 (2006).

[13] Vakoc, B. J., Shishko, M., Yun, S. H., Oh, W.Y., Suter, M. J., Desjardins, A. E., Evans, J. A., Nishioka, N. S., Tearney, G. J. and Bouma, B. E., "Comprehensive esophageal microscopy by using optical frequency-domain imaging (with video)," Gastrointest. Endosc. 65(6), 898-905 (2007).

[14] Yang, J. M., Maslov, K., Yang, H. C., Zhou, Q., Shung, K. K. and Wang, L. V., "Photoacoustic endoscopy," Opt. Lett. 34(10), 1591-1593 (2009).

[15] Yang, J. M., Maslov, K., Yang, H. C., Zhou, Q. and Wang, L. V., "Endoscopic photoacoustic microscopy," Proc. SPIE 7177, 71770N (2009).

[16] Oraevsky, A. A. and Karabutov, A. A., "Optoacoustic Tomography," [Biomedical Photonics Handbook], CRC Press, New York, 34 (2003).

[17] Wang, L. V., "Multiscale photoacoustic microscopy and computed tomography," Nat. Photonics 3, 503-509 (2009).

[18] Emelianov, S. Y., Li, P.-C. and O'Donnell, M., "Photoacoustics for molecular imaging and therapy," Phys. Today 62(5), 34-39 (2009).

[19] Ntziachristos, V. and Razansky, D., "Molecular imaging by means of multispectral optoacoustic tomography (MSOT)," Chem. Rev. 110(5), 2783-2794 (2010).

[20] Beard, P., "Biomedical photoacoustic imaging," Interface Focus 1, 602-631 (2011).

[21] Wang, L. V. and Hu, S., "Photoacoustic tomography: in vivo imaging from organelles to organs," Science 335(6075), 1458-1462 (2012).

[22] Cox, B., Laufer, J. G., Arridge, S. R. and Beard, P. C., "Quantitative spectroscopic photoacoustic imaging: a review," J. Biomed. Opt. 17(6), 061202 (2012).

[23] Galanzha, E. I. and Zharov, V. P., "Circulating tumor cell detection and capture by photoacoustic flow cytometry in vivo and ex vivo," Cancers 5, 1691-1738 (2013).

[24]Zhang, H. F., Maslov, K., Stoica, G. and Wang, L. V., "Functional photoacoustic microscopy for high-resolution and noninvasive in vivo imaging," Nat. Biotechnol. 24(7), 848-851 (2006).

[25] Shah, J., Park, S., Aglyamov, S., Larson, T., Ma, L., Sokolov, K., Johnston, K., Milner, T. and Emelianov, S. Y., "Photoacoustic imaging and temperature measurement for photothermal cancer therapy," J. Biomed. Opt. 13(3), 034024 (2008).

[26]Favazza, C. P., Cornelius, L. A. and Wang, L. V., "In vivo functional photoacoustic microscopy of cutaneous microvasculature in human skin," J. Biomed. Opt. 16, 026004 (2011).

[27] Allen, T. J., Hall, A., Dhillon, A. P., Owen, J. S. and Beard, P. C., "Spectroscopic photoacoustic imaging of lipidrich plaques in the human aorta in the 740 to $1400 \mathrm{~nm}$ wavelength range," J. Biomed. Opt. 17, 061209 (2012).

[28] Yao, J., Huang, C. H., Wang, L., Yang, J. M., Gao, L., Maslov, K. I., Zou, J. and Wang, L. V., "Wide-field fastscanning photoacoustic microscopy based on a water-immersible MEMS scanning mirror," J. Biomed. Opt. 17(8), 080505 (2012).

[29] Laufer, J., Johnson, P., Zhang, E., Treeby, B., Cox, B., Pedley, B. and Beard, P., "In vivo preclinical photoacoustic imaging of tumor vasculature development and therapy," J. Biomed. Opt. 17(5), 056016 (2012).

[30] Kruger, R. A., Kuzmiak, C. M., Lam, R. B., Reinecke, D. R., Del Rio, S. P. and Steed, D., "Dedicated 3D photoacoustic breast imaging," Med Phys. 40(11), 113301 (2013). 
[31] Nasiriavanaki, M., Xia, J., Wan, H., Bauer, A. Q., Culver, J. P. and Wang, L. V., "High-resolution photoacoustic tomography of resting-state functional connectivity in the mouse brain," Proc. Natl. Acad. Sci. U. S. A. 111(1), $21-26$ (2014).

[32] Oraevsky, A. A., Esenaliev, R. O. and Karabutov, A., "Laser optoacoustic tomography of layered tissues: signal processing," Proc. SPIE 2979, 59-70 (1997).

[33] Viator, J. A, Paltauf, G., Jacques, S. L. and Prahl, S. A., "Design and testing of an endoscopic photoacoustic probe for determination of treatment depth after photodynamic therapy," Proc. SPIE 4256, 16-27 (2001).

[34] Sethuraman, S., Aglyamov, S. R., Amirian, J. H., Smalling, R. W. and Emelianov, S. Y., "Intravascular photoacoustic imaging using an IVUS imaging catheter," IEEE Trans. Ultrason. Ferroelectr. Freq. Control. 54(5), 978-986 (2007).

[35] Wang, B., Su, J. L., Karpiouk, A. B., Sokolov, K. V., Smalling, R. W. and Emelianov, S. Y., "Intravascular photoacoustic imaging," IEEE J. Sel. Top. Quantum Electron. 16, 588-599 (2010).

[36] Karpiouk, A. B., Wang, B. and Emelianov, S. Y., "Development of a catheter for combined intravascular ultrasound and photoacoustic imaging," Rev. Sci. Instrum. 81, 014901 (2010).

[37] Wang, X., Roberts, W. W., Carson, P. L., Wood, D. P. and Fowlkes, J. B., "Photoacoustic tomography: a potential new tool for prostate cancer," Biomed. Opt. Express 1, 1117-1126 (2010).

[38] Yaseen, M. A., Ermilov, S. A., Brecht, H. P., Su, R., Conjusteau, A., Fronheiser, M., Bell, B. A., Motamedi, M. and Oraevsky, A. A., "Optoacoustic imaging of the prostate: development toward image-guided biopsy," J. Biomed. Opt. 15, 021310 (2010).

[39] Yang, Y., Li, X., Wang, T., Kumavor, P. D., Aguirre, A., Shung, K. K., Zhou, Q., Sanders, M., Brewer, M. and $\mathrm{Zhu}, \mathrm{Q}$., "Integrated optical coherence tomography, ultrasound and photoacoustic imaging for ovarian tissue characterization,” Biomed. Opt. Express 2, 2551-2561 (2011).

[40] Jansen, K., van der Steen, A. F., van Beusekom, H. M., Oosterhuis, J. W. and van Soest, G., "Intravascular photoacoustic imaging of human coronary atherosclerosis," Opt. Lett. 36, 597-599 (2011).

[41]Zhang, E. Z. and Beard, P. C., “A miniature all-optical photoacoustic imaging probe,” Proc. SPIE 7899, 78991F (2011).

[42] Wang, B., Karpiouk, A., Yeager, D., Amirian, J., Litovsky, S., Smalling, R. and Emelianov, S., "Intravascular photoacoustic imaging of lipid in atherosclerotic plaques in the presence of luminal blood," Opt. Lett. 37, 12441246 (2012).

[43] Karpiouk, A. B., Wang, B., Amirian, J., Smalling, R. W. and Emelianov, S. Y., "Feasibility of in vivo intravascular photoacoustic imaging using integrated ultrasound and photoacoustic imaging catheter,” J. Biomed. Opt. 17(9), 096008 (2012).

[44] Yang, J. M., Favazza, C., Chen, R., Maslov, K., Cai, X., Zhou, Q., Shung, K. K. and Wang, L. V., "Volumetric photoacoustic endoscopy of upper gastrointestinal tract: ultrasonic transducer technology development," Proc. SPIE 7899, 78990D (2011).

[45] Yang, J. M., Favazza, C., Chen, R., Yao, J., Cai, X., Maslov, K., Zhou, Q., Shung, K. K. and Wang, L. V., "Simultaneous functional photoacoustic and ultrasonic endoscopy of internal organs in vivo," Nat. Med. 18(8), 1297-1302 (2012).

[46] Yang, J. M., Favazza, C., Chen, R., Yao, J., Cai, X., Maslov, K., Zhou, Q., Shung, K. K. and Wang, L. V., “Toward dual-wavelength functional photoacoustic endoscopy: laser and peripheral optical systems development," Proc. SPIE 8223, 822316 (2012).

[47] Yang, J. M., Chen, R., Favazza, C., Yao, J., Li, C., Hu, Z., Zhou, Q., Shung, K. K. and Wang, L. V., “A 2.5-mm diameter probe for photoacoustic and ultrasonic endoscopy," Opt. Express 20, 23944-23953 (2012).

[48] Li, C., Yang, J. M., Chen, R., Zhang, Y., Xia, Y., Zhou, Q., Shung, K. K. and Wang, L. V., "Photoacoustic endoscopic imaging study of melanoma tumor growth in a rat colorectum in vivo," Proc. SPIE 8581, 85810D (2013).

[49] Li, C., Yang, J. M., Chen, R., Yeh, C.-H., Zhu, L., Maslov, K., Zhou, Q., Shung, K. K. and Wang, L. V., "Urogenital photoacoustic endoscope," Opt. Lett. 39(6), 1473-1476 (2014).

[50] Yang, J. M., Li, C., Chen, R., Zhou, Q., Shung, K. K. and Wang, L. V., "Catheter-based photoacoustic endoscope," J. Biomed. Opt. 19(6), 066001 (2014).

[51] Menzel, J. and Domschke, W., "Gastrointestinal miniprobe sonography: the current status,” Am. J. Gastroenterol. 95, 605-616 (2000).

[52] Tearney, G. J., Brezinski, M. E., Bouma, B. E., Boppart, S. A., Pitris, C., Southern, J. F. and Fujimoto, J. G., “In vivo endoscopic optical biopsy with optical coherence tomography," Science 276, 2037-2039 (1997). 
[53] Yaqoob, Z., Wu, J., McDowell, E. J., Heng, X. and Yang, C., "Methods and application areas of endoscopic optical coherence tomography,” J. Biomed. Opt. 11, 063001 (2006).

[54] Fu, H. L., Leng, Y., Cobb, M. J., Hsu, K., Hwang, J. H. and Li, X., "Flexible miniature compound lens design for high-resolution optical coherence tomography balloon imaging catheter," J. Biomed. Opt. 13(6), 060502 (2008).

[55] Gora, M. J., Sauk, J. S., Carruth, R. W., Gallagher, K. A., Suter, M. J., Nishioka, N. S., Kava, L. E., Rosenberg, M., Bouma, B. E. and Tearney, G. J., "Tethered capsule endomicroscopy enables less invasive imaging of gastrointestinal tract microstructure," Nat. Med. 19, 238-240 (2013).

[56] Cannata, J. M., Ritter, T. A., Chen, W. H., Silverman, R. H. and Shung, K. K., "Design of efficient, broadband single-element (20-80 MHz) ultrasonic transducers for medical imaging applications," IEEE Trans. Ultrason. Ferroelectr. Freq. Control. 50, 1548-1557 (2003).

[57] Wang, L. H., Jacques, S. L. and Zheng, L. Q., "MCML-Monte Carlo modeling of photon transport in multi-layered tissues," Comput. Meth. Programs Biomed. 47, 131-146 (1995).

[58] Bashkatov, A. N., Genina, E. A., Kochubey, V. I. and Tuchin, V. V., "Optical properties of human skin, subcutaneous and mucous tissues in the wavelength range from 400 to $2000 \mathrm{~nm}$," J. Phys. D: Appl. Phys. 38, 25432555 (2005).

[59] Laser Institute of America, [American National Standard for Safe Use of Lasers (ANSI Z136.1-2007)], American National Standards Institute, New York (2007).

[60] Yang, J. M., Li, C., Chen, R., Rao, B., Yao, J., Yeh, C. H., Danielli, A., Maslov, K., Zhou, Q., Shung, K. K. and Wang, L. V., "Optical-resolution photoacoustic endomicroscopy in vivo," Biomed. Opt. Express 6(3), 918-932 (2015). 